\title{
Usages et usagers des bibliothèques dans le Haut- Empire romain
}

\section{Marie Dallies}

\section{(2) OpenEdition}

1 Journals

Édition électronique

URL : http://journals.openedition.org/rbnu/283

DOI : 10.4000/rbnu.283

ISSN : 2679-6104

Éditeur

Bibliothèque nationale et universitaire de Strasbourg

\section{Édition imprimée}

Date de publication : 23 mai 2018

Pagination : 8-15

ISSN : 2109-2761

\section{Référence électronique}

Marie Dallies, «Usages et usagers des bibliothèques dans le Haut-Empire romain », La Revue de la BNU [En ligne], 17 | 2018, mis en ligne le 05 septembre 2018, consulté le 10 décembre 2020. URL : http:// journals.openedition.org/rbnu/283 ; DOI : https://doi.org/10.4000/rbnu.283

\section{(c) (i) (5)}

La Revue de la BNU est mise à disposition selon les termes de la Licence Creative Commons Attribution - Pas d'Utilisation Commerciale - Partage dans les Mêmes Conditions 4.0 International. 


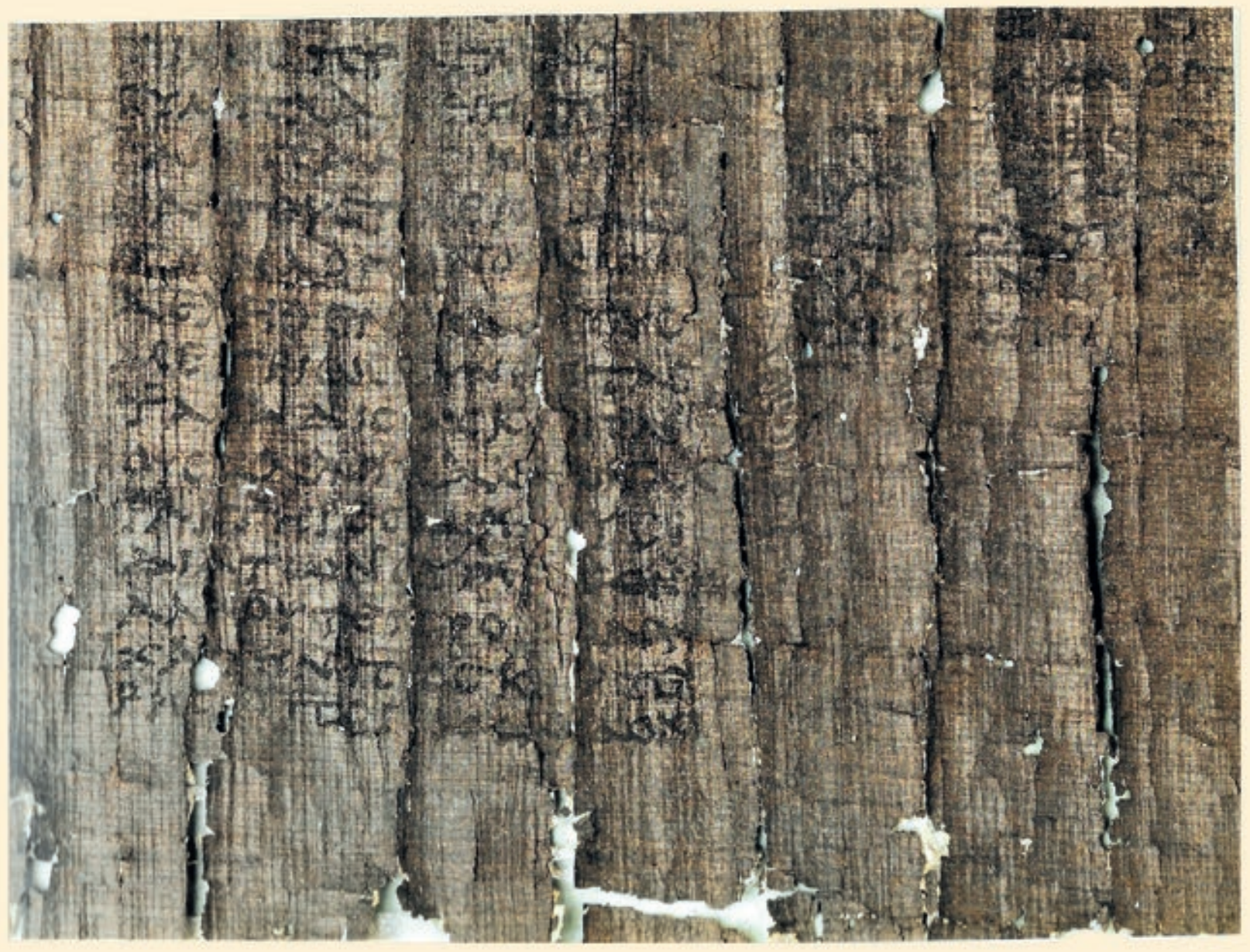

Manuscrit du De Rhetorica I de Philodème de Gadara, conservé à Naples,

Biblioteca Nazionale Vittorio Emanuele III, Centro Internazionale per lo Studio dei Papiri Ercolanesi (PHerc. 1427, cadre 2), provenant de la villa des Papyrus [reproduit dans Guidobaldi, M. P. et Esposito, D., Herculanum, photographies de L. Pedicini, trad. de D. A. Canal, Paris, Imprimerie nationale, 2012, p. 91]. 


\section{USAGES ET USAGERS DES BIBLIOTHÈQUES DANS LE HAUT-EMPIRE ROMAIN \\ PAR MARIE DALLIES}

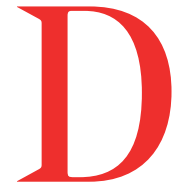

ès l'époque républicaine, la bibliothèque, dans l'habitat des couches aisées de la société romaine, constitue une pièce incontournable de la maison, dont l'emplacement doit être stratégiquement choisi afin d'assurer la bonne conservation des livres, comme le précise Vitruve à plusieurs reprises dans son traité $D e$ l'architecture ${ }^{1}$. La bibliothèque, en effet, est un marqueur du rang social et Sénèque ne peut que déplorer l'ostentation dont font preuve les parvenus et fanfarons, en exposant les œuvres complètes des historiens et orateurs qu'ils n'ont probablement pas lues ${ }^{2}$. L'importance de cette pièce se traduit notamment par l'évocation plus ou moins détaillée que les lettrés font de leur bibliothèque personnelle ou de celles qu'ils fréquentent chez leurs amis : Cicéron dans sa Correspondance évoque à plusieurs reprises les livres qu'il emprunte à plusieurs de ses amis, notamment Atticus, qui accueille volontiers chez lui des lettrés et de très bons lecteurs, et donne des conseils à Cicéron pour l'aménagement de sa bibliothèque ${ }^{3}$. Les bibliothèques privées constituent donc un lieu d'échanges et de fréquentation entre membres de l'aristocratie, et sous la République, trois d'entre elles sont particulièrement éminentes : celle des Scipion, réunie par les fils de Paul-Émile après la victoire de ce dernier sur Persée, roi de Macédoine, à Pydna en 168 av. J.-C., celle de Faustus Sylla, rapportée par le dictateur lors de la prise d'Athènes en 86 av. J.-C. et transmise à son fils, celle de Lucullus enfin, que le général rassembla lors de la troisième guerre mithridatique $^{4}$. Les collections, formées à partir de pillages lors de la conquête, offrent donc l'accès à des textes parfois peu accessibles : la bibliothèque de Sylla, par exemple, est constituée des œuvres d'Aristote et de Théophraste, très peu répandues à cette époque en Italie. On comprend ainsi l'intérêt qu'elles suscitent, surtout quand leur propriétaire les ouvre à un cercle plus large que son entourage : au témoignage de Plutarque, Lucullus permet l'accès de sa bibliothèque à tous et accueille notamment les Grecs, installés à Rome ou seulement de passage ; les visiteurs peuvent y emprunter des livres, mais aussi y étudier, puisqu'elle possède des galeries et des salles de travail dédiées aux lecteurs. Même si l'on n'a pas de témoignage archéologique concernant la bibliothèque de Lucullus, celle retrouvée dans la villa des Papyrus à Herculanum ${ }^{5}$ peut nous donner une idée de son organisation. Les fouilles menées au $18^{\mathrm{e}}$ siècle ont mis en évidence une série de quatre pièces, contenant près d'un millier de "uolumina ", disposés sur des niches creusées dans les murs, entreposés en piles par terre ou conservés à la verticale dans des boîtes à rouleaux. Si l'on compte une centaine de rouleaux en langue latine, l'essentiel de la collection est en langue grecque et est constitué presque exclusivement d'œuvres philosophiques, en majorité relevant de l'épicurisme. On y trouve notamment plusieurs livres de La nature d'Épicure, ainsi qu'une grande partie des œuvres de Philodème de Gadara, épigone d'Épicure très lié à plusieurs membres de l'aristocratie romaine et qui a pu séjourner 
et travailler dans la villa. La présence, dans ces pièces, de plusieurs petits bustes de bronze (Épicure, Zénon, Démosthène notamment) laisse penser qu'ils étaient placés dans les niches, pour signaler les ouvrages des auteurs portraiturés : ce détail, facilitant le repérage dans la bibliothèque, tendrait à appuyer l'hypothèse qu'elle était sans doute ouverte à un public plus large que le proche entourage du maitre de maison, comme c'était le cas pour la bibliothèque de Lucullus. Les bibliothèques privées, dans la Rome et l'Italie républicaines, peuvent donc, quand leurs propriétaires le permettent voire l'encouragent, faciliter la diffusion et l'échange des œuvres, pratique d'autant plus remarquable que ces quelques bibliothèques dont le souvenir nous est resté étaient majoritairement composées d'œuvres grecques et hellénistiques, encore assez peu répandues dans cette région. Elles jouent donc un rôle non négligeable dans le développement du philhellénisme à Rome et dans la transmission des savoirs.

Il existait pourtant, depuis au moins le $3^{\mathrm{e}}$ siècle av. J.-C., des bibliothèques publiques ; mais celles-ci se situent dans la partie orientale de l'Empire romain. Généralement adossées à un "gymnasium », elles se développent dans d'assez nombreuses cités (Athènes, bien sûr, ainsi que des cités comme Cos ou Rhodes), mais les deux plus célèbres demeurent les bibliothèques d'Alexandrie et de Pergame ${ }^{6}$. La première, fondée en 300 à l'instigation des Ptolémée, abrite, au moment où César arrive dans la cité en 48 , près de sept cent mille ouvrages, tous écrits en grec : la politique était de faire traduire toute œuvre, rédigée dans une autre langue, qui transitait par Alexandrie. La bibliothèque de Pergame, quant à elle, est moins richement dotée (environ deux cent mille ouvrages au milieu du $1^{\text {er }}$ siècle av. J.-C.), mais sa rivalité avec la bibliothèque d'Alexandrie, et le refus de cette dernière d'exporter du papyrus, ont permis l'invention du parchemin pour remédier à la pénurie.

On peut supposer que l'exemple de ces réussites ne fut pas étranger au désir, manifesté d'abord par César puis, à sa suite, par plusieurs empereurs, de créer et d'entretenir à Rome plusieurs bibliothèques publiques. Ces établissements apparaissent dans la Ville à la fin du $1^{\text {er }}$ siècle av. J.-C., puisqu'entre 39 et 28, Asinius Pollion, concrétisant le projet césarien, installe dans l'« Atrium Libertatis " une bibliothèque ouverte à tous. En 28 toujours, Auguste dédicace sur le Palatin le temple d'Apollon, qui abrite également une bibliothèque, et inaugure une vingtaine d'années plus tard la bibliothèque du "porticus Octauiae ", au sud du Champ de Mars. Tibère poursuit l'œuvre de son prédécesseur en supervisant la construction de deux bibliothèques publiques : l'une dans le temple $\mathrm{du}$ divin Auguste, au pied du Palatin, l'autre dans la "domus Tiberiana », résidence impériale sise sur le Palatin. Il faut ensuite attendre le règne de Vespasien pour voir l'inauguration d'une nouvelle bibliothèque publique, celle du «templum Pacis », dédicacé en 75 pour commémorer la victoire sur les Juifs et la prise du temple de Jérusalem. Trajan consacre de part et d'autre de la colonne Trajane deux constructions adossées à la basilique ulpienne, qui abritent une bibliothèque. Enfin, à la toute fin du Haut-Empire, sous le règne de Sévère Alexandre, Iulius Africanus mentionne dans les Cestes qu'il a établi les plans d'une bibliothèque à l'est du Panthéon, près des termes d'Alexandre, sur le Champ de Mars : huit édifices de ce genre apparaissent donc à Rome entre la fin du $1^{\text {er }}$ siècle av. J.-C. et le milieu $\mathrm{du} 3^{\mathrm{e}}$ siècle après J.-C., la plupart étant adossées à un monument religieux, sans doute parce que les temples faisaient souvent office de dépôts d'archives. Toutes ces bibliothèques sont construites sur le même plan : elles abritent des collections grecques et latines (la présence des deux salles pour la bibliothèque ulpienne révèle d'ailleurs architecturalement cette répartition), identifiées par la présence de statues des auteurs, destinées tant à faciliter le repérage qu'à célébrer les grandes figures grecques et romaines.

Parallèlement à ces édifications impériales, on peut noter le développement, durant l'Empire et dans tout le bassin méditerranéen, de monuments fondés grâce à l'évergétisme d'un particulier, qui dédie à sa ville un édifice culturel, avec les collections et les statues qui seront utiles à sa décoration et au repérage des ouvrages. Dans la péninsule italienne, Pline le Jeune fait construire à Côme, sa cité natale, une bibliothèque, dont l'ouverture est motivée par la volonté de faciliter l'éducation des enfants de la cité ; Matidia, tante par alliance d'Antonin le Pieux, fait élever un bâtiment de ce type à Suessa Aurunca, cité avec laquelle elle entretient des liens étroits ; à Volsinii, un ancien primipile 


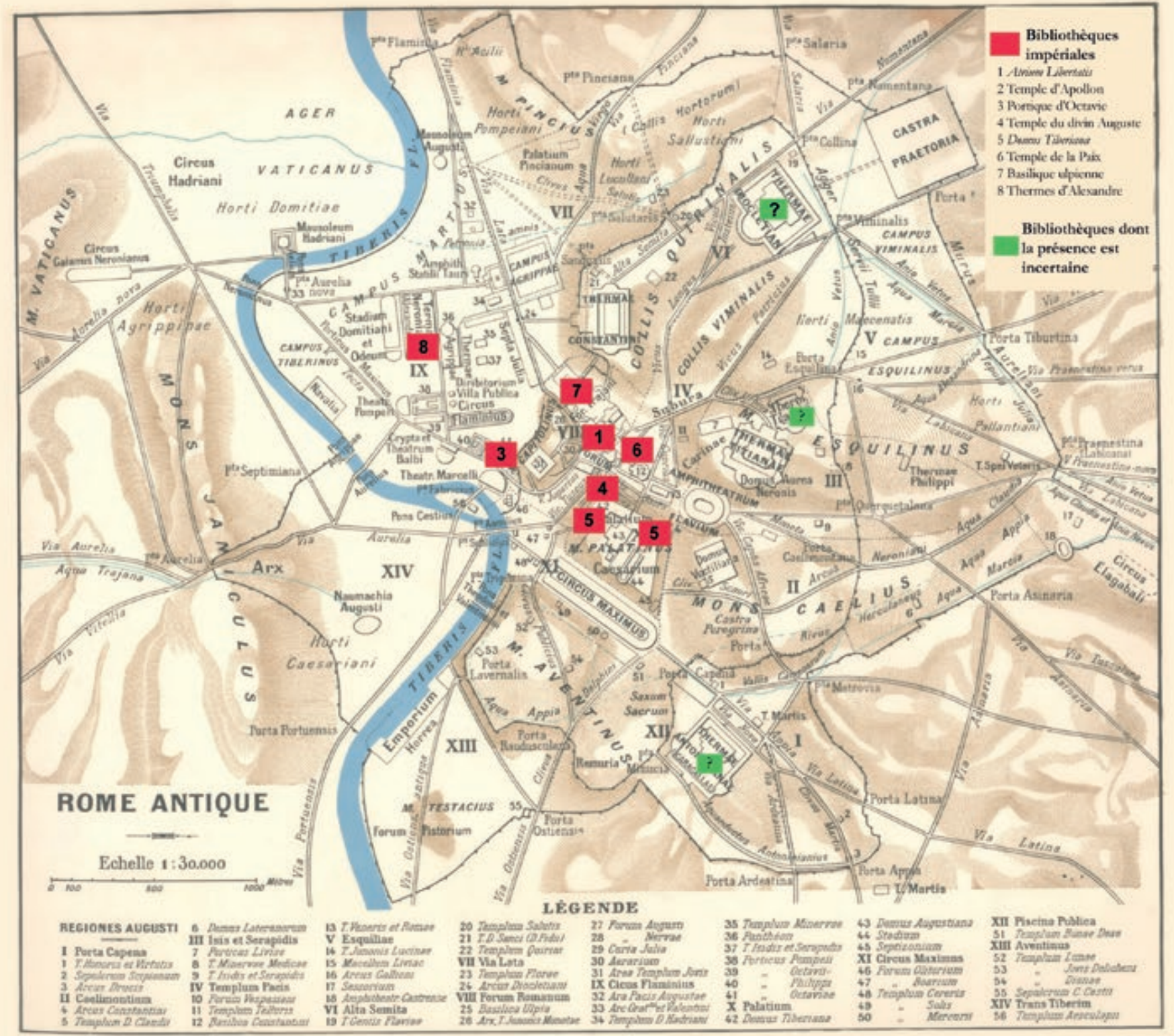

Plan des bibliothèques publiques romaines

(fond de carte issu du Nouveau Larousse illustré, éd. 1866-1877) 


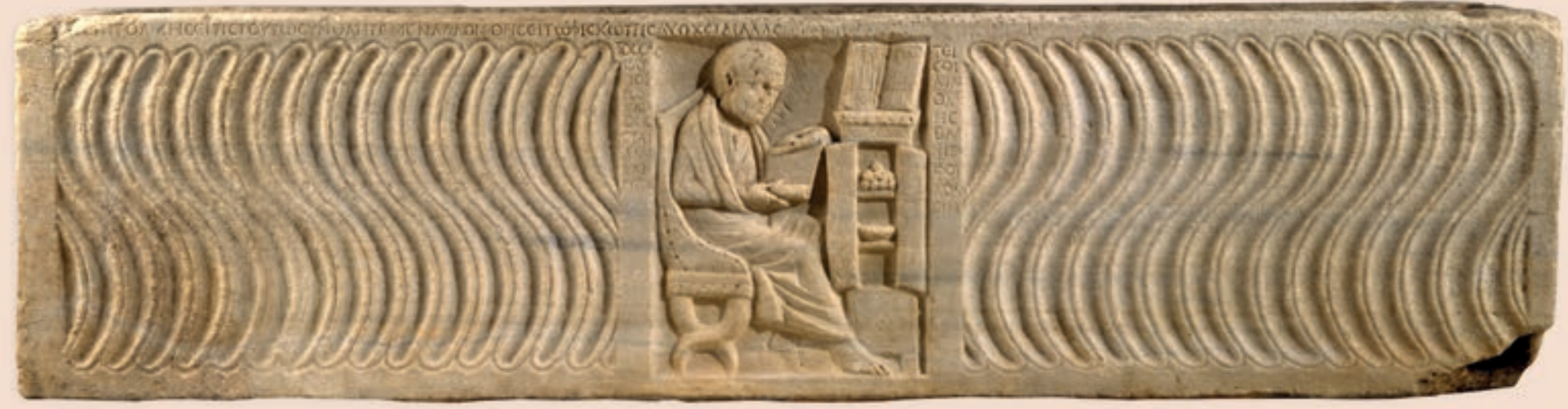

Bas-relief d'un sarcophage du $4^{e}$ siècle, montrant un médecin lisant devant un armarium (coll. New York, The Metropolitan Museum of Art) 
fait don, à sa mort, d'une bibliothèque à sa cité, tandis que Tibur est dotée d'une bibliothèque située dans le temple d'Hercule. Cet évergétisme existe également dans le reste de l'Empire, par exemple à Timgad, au $3^{\mathrm{e}}$ siècle, sous l'impulsion du sénateur Iulius Quintianus Flavius Rogatianus, à Athènes, où deux bibliothèques sont construites, l'une à l'initiative d'un particulier, Pantainos, sous le règne de Trajan, l'autre grâce à l'évergétisme d'Hadrien, à Pergame, où Flavia Métiline offre une bibliothèque au sanctuaire d'Asclépios, à Éphèse enfin, où la bibliothèque dédiée à Celsus Polemaneus par son fils en fait le troisième monument de ce type le plus important, après ceux d'Alexandrie et de Pergame $^{7}$. Ces quelques exemples peuvent sembler bien parcellaires au vu de l'étendue de l'Empire romain : ils nous incitent cependant à penser que les bibliothèques publiques se développent dans tout l'Empire, au moins dans les villes importantes, mais aussi, probablement, dans un certain nombre de cités de taille plus modeste.

Enfin, même si leur présence a souvent été considérée comme acquise, il faut se montrer prudents quant à l'existence de bibliothèques dans les établissements thermaux. L'hypothèse a été fondée sur l'existence de salles, notamment dans les thermes de Trajan et de Caracalla à Rome, possédant sur certains murs deux rangées de niches et sur la mention, dans l'Histoire Auguste, du déménagement de la bibliothèque ulpienne dans les thermes de Dioclétien ${ }^{8}$. Mais les travaux récents de T. Dix et de G. Houston ${ }^{9}$ incitent à leur attribuer d'autres fonctions : les deux exèdres des thermes de Trajan seraient plutôt une salle de lecture et de "recitationes ", tandis que la démesure des salles et des niches dans les thermes de Caracalla laisse penser qu'il ne s'agit pas d'une bibliothèque, sans qu'il soit pour autant possible d'attribuer une fonction précise à ces pièces.

La localisation des bibliothèques publiques à Rome montre une concentration de ces édifices dans des lieux symboliques du pouvoir impérial - que ce soit sur le Palatin, colline des résidences impériales, ou sur le forum que les Princes se font construire. Ils constituent, en premier lieu, la marque d'une ambition culturelle : les empereurs qui n'ont pas fait édifier de bibliothèques ne se sont pas nécessairement désintéressés de cette question, loin de là. Il convenait en effet d'assurer l'entretien de ces bâtiments, particulièrement vulnérables au feu. On peut ainsi mentionner l'entreprise de reconstruction menée par Domitien, qui veille notamment à la réfection des bibliothèques du temple du divin Auguste et du portique d'Octavie, et fait venir depuis Alexandrie des copies des ouvrages disparus ${ }^{10}$, ou celle assurée par Septime Sévère et Caracalla en faveur, à nouveau, de la bibliothèque du Portique d'Octavie, et peut-être aussi en faveur de celles du temple de la Paix et du Palatin, détruites dans un incendie en $192^{11}$. Mais l'intérêt des Princes pour les bibliothèques comporte aussi une dimension politique, puisqu'ils ont un droit de regard sur les collections - on peut ainsi songer à la censure qu'Auguste fait peser sur les ouvrages d'Ovide en raison de son bannissement ${ }^{12}$, ou aux autodafés prononcés par Auguste, Tibère, Caligula, Néron ou Domitien contre les œuvres d'opposants politiques $^{13}$. À l'inverse, les empereurs peuvent promouvoir certains auteurs qu'ils apprécient : Tibère fait entrer dans les bibliothèques publiques les œuvres des poètes grecs Euphorion de Chalcis, Rhianos de Crète et Parthenios de Nicée, qu'il admire tout particulièrement ${ }^{14}$. On ne connaît pas précisément l'état des collections des différentes bibliothèques romaines, mais au témoignage d'Ovide et de Suétone ${ }^{15}$, elles abritent des ouvrages à la fois d'auteurs anciens et de contemporains. On peut supposer sans trop de difficultés que l'on y trouvait les œuvres de référence et que chaque bibliothèque tendait à se spécialiser dans un domaine.

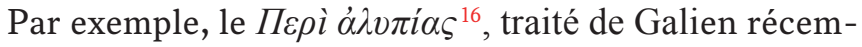
ment découvert, nous apprend que les bibliothèques du Palatin possédaient un fond spécialisé dans le domaine scientifique et médical : Galien déplore la perte des ouvrages philosophiques et médicaux auxquels il avait notamment apporté d'importantes modifications graphiques. Quelques mentions de recherches bibliographiques rapportées par Aulu-Gelle dans les Nuits attiques laissent penser que la bibliothèque du temple de la Paix regroupait plutôt les ouvrages consacrés à la grammaire et à la philologie, tandis qu'il consulte dans la bibliothèque ulpienne les édits des préteurs : soit celle-ci s'est spécialisée dans le domaine juridique, soit elle constitue un centre de dépôt des archives impériales, comme c'est le cas aussi des bibliothèques du Palatin ${ }^{17}$. Ces indications demeurent fragmentaires, mais elles permettent de supposer que les bibliothèques 
publiques romaines, sur un territoire relativement réduit, offraient une certaine complémentarité dans leurs collections.

Quant à l'organisation de ces bibliothèques, elle est très hiérarchisée ${ }^{18}$. À leur tête se trouve un chevalier, de rang procuratorien (le titre de " procurator bibliothecarum " apparaît dans les années 120 après J.-C.), qui supervise l'ensemble des bibliothèques et joue un rôle de conseiller, notamment sur la tenue des collections, auprès du Prince. Il est également le supérieur des directeurs de chaque bibliothèque, qui peuvent eux-mêmes être procurateurs, mais d'un rang inférieur, ou des esclaves appelés " uilici », chargés d'une section (grecque ou latine) de la bibliothèque et responsables d'un groupe d'esclaves " a bybliotheca " préposés à des tâches diverses - copier ou corriger des manuscrits, les restaurer, localiser un ouvrage, tenir le catalogue, apporter un uolumen au lecteur, etc. Ce dernier n'est en effet pas autorisé à aller se servir tout seul : c'est un membre du personnel qui doit lui amener l'ouvrage qu'il souhaite consulter ${ }^{19}$. D'ailleurs, les niches contenant les rouleaux sont souvent séparées du sol par un podium, comme le montre l'exemple de la bibliothèque d'Éphèse, et celles des niveaux supérieurs ne sont accessibles qu'à l'aide d'échelles. Ces mesures visaient sans doute à assurer la conservation des «uolumina», d'un maniement d'autant plus fragile qu'ils étaient généralement empilés, à l'intérieur des niches, dans des armoires (" armaria ») et transportés, ensuite, dans des coffrets cylindriques (appelés " capsae » ou « scrinia »).

On possède, enfin, quelques indications éparses sur les conditions d'accès des lecteurs aux bibliothèques. Une inscription ${ }^{20}$ retrouvée dans le bâtiment rappelle deux règles en vigueur dans la bibliothèque de Pantainos, à Athènes, à propos des horaires d'ouverture et de l'emprunt. L'édifice est ouvert de la première à la sixième heure, soit, approximativement, de l'aurore à la mi-journée. Le reste du temps, l'accès est fermé par une grille ou une porte, comme en témoignent les traces archéologiques de la bibliothèque ulpienne ou de celle de Timgad. Le règlement de la bibliothèque de Pantainos précise également qu'il est interdit d'emporter un ouvrage hors du bâtiment : il n'est donc pas possible d'emprunter, seulement de consulter. On a vu précédemment, à travers l'exemple de Galien, qu'il est cependant autorisé d'annoter et de corriger les manuscrits que l'on examine et de contribuer ainsi à la révision et à l'amélioration des textes proposés. Il semblerait que la question de l'emprunt soit propre à chaque bibliothèque : une lettre ${ }^{21} \mathrm{du}$ futur Marc Aurèle à Fronton, son maître de rhétorique, l'informe qu'il lui faudra « courtiser " (subigitare) le bibliothécaire de la « domus Tiberiana " pour emprunter l'ouvrage de Caton que lui-même a pris dans la bibliothèque du temple d'Apollon. Cela peut signifier soit que ce bâtiment est réservé à un cercle étroit autour de la famille impériale, soit que le prêt n'y est pas habituellement autorisé. À l'inverse, Aulu-Gelle, dans une anecdote, mentionne qu'un membre du groupe d'amis constitués de rhéteurs et de philosophes, qu'il fréquente l'été à Tibur, emprunte à la bibliothèque de la ville un ouvrage d'Aristote pour finir de convaincre ses compagnons du bien-fondé de sa démonstration ${ }^{22}$. Ce dernier exemple rappelle que l'essentiel du public des bibliothèques de l'empire romain est constitué de lettrés : les exemples que l'on possède sont ceux des écrivains eux-mêmes ou des érudits qu'ils fréquentent, dans tous les domaines - l'exemple de Galien souligne que les médecins pouvaient fréquemment y venir. Enfin, il ne faut pas oublier le public estudiantin : les exemples d'Aulu-Gelle, du futur Marc Aurèle, de Pline le Jeune, qui adosse la bibliothèque qu'il fait construire à Côme à l'école de rhétorique qu'il fonde en même temps dans sa cité, nous apprennent que les bibliothèques constituent pour les jeunes gens de l'élite romaine un lieu privilégié pour approfondir les connaissances qu'il acquièrent auprès de leurs maîtres.

Malgré le nombre relativement faible d'édifices attestés et des sources qui demeurent assez parcellaires sur la question, on peut se rendre compte du développement et de l'importance que les bibliothèques prennent sous l'Empire romain. Ouvertes à tous, peut-être gratuitement (il n'existe à ce jour aucun témoignage sur l'existence de frais d'inscription) grâce à la générosité des Princes ou de particuliers, elles favorisent la diffusion des œuvres grecques et romaines dans tout l'Empire et constituent ainsi un vecteur de circulation des savoirs. 


\section{ORIENTATIONS BIBLIOGRAPHIQUES}

- Casson, L., Libraries in the Ancient World, New Haven, Yale University Press, 2001

- Dix, T. K. et Houston, G. W., « Public Libraries in the City of Rome: from the Augustan Age to the Diocletian Time ", in MEFRA 118 (2), 2006, p. 671-717

- Houston, G. W., Inside Roman Libraries, Chapel Hill, The University of North Carolina Press, 2017

- Perrin, Y. (dir.), Neronia VIII. Bibliothèques, livres et culture écrite dans l'empire romain de César à Hadrien, Bruxelles, Latomus, 2010

- Strocka, V., « Römische Bibliotheken », in Gymnasium 88, 1981, p. $298-329$

\section{NOTES}

1- Cf. Vitr. I, 2, 7 ; VI, 4, 1; 5, 2.

2- Cf. Sén., Tranq. 9, 4-7, qui souligne que la bibliothèque est devenue un « ornement obligé de la maison " (necessarium domus ornamentum). Il vise des personnes telles que Pétrone les a dépeintes sous les traits de Trimalcion (Petr. 48, 4), heureux propriétaire de « trois bibliothèques, dont une latine et une grecque ", mais incapable de se rendre compte de l'absurdité de ses propos!

3- Cf. Cic., Att. II, 1, 12 ; 3, 4 ; IV, 10, 1 ; Fin. III, 7-8 ; Nep., Att. 13, 3. On connaît par ailleurs les bibliothèques de Pline le Jeune dans sa villa des Laurentes (Plin., Epist. II, 17), de Perse (Prob., Vita Pers. 7), de Martial (Mart. XIV, 190) ou encore de Pontianus, camarade d'études d'Apulée (Apul., Apol. 55, 3).

4- À propos de ces trois bibliothèques, cf. Plut., Aem. 28, 11 ; Sul. 26, 1-3; Lucul. 42, 12. On peut noter que Cicéron fréquenta particulièrement les deux dernières (cf. Cic, Att. IV, 10, 1 ; Fin. III, 78).

5- Nous renvoyons, à ce propos, à deux ouvrages récents synthétisant les découvertes sur cet édifice : Delattre, D., La villa des Papyrus et les rouleaux d'Herculanum : la bibliothèque de Philodème, Liège, CEDOPAL, 2006 ; Houston, G., Roman Libraries, p. 87-129.

6- Pour plus de détails sur toutes ces bibliothèques, nous renvoyons aux ouvrages de Canfora, L., La véritable histoire de la bibliothèque d'Alexandrie, trad. de l'italien par J.-P. Manganaro et D. Dubroca, Paris, Desjonquères, 1986 ; Casson, L., Libraries, p. 31-53.

7- À propos de la bibliothèque de Côme : Plin., Epist. I, 8 ; CIL V, $5262=$ CIL VI, p. $4712=I L S$ 2927. À Suessa Aurunca : CIL X, $4760=$ ILS 6296. À Volsinii : CIL XI, 2704 b. À Tibur : Gell. IX, 14, 3 et XIX, 5, 4. À Timgad : $A E$ 1908, $12=I L S$ 9362. À Athènes : $S E G$ XXI, 703 = Agora I, 848 ; Paus. I, 18, 9. À Pergame : AvP VIII, 3, $\mathrm{n}^{\circ}$ 38. À Ephèse : Keil, J., Forschungen in Ephesos, 1923, p. 61-62, $\mathrm{n}^{\circ} 1$, p. $175, \mathrm{n}^{\circ} 13$.

8- Hist. Aug., Prob. 2,1

9- Dix, T., Houston, G., Public Libraries p. 701-706

10- Cf. Suet., Dom. 20, 1 ; Mart. XII, 3, 78 ; D.C. LXVI, 24, 2.

11- À propos du portique d'Octavie, cf. CIL VI, 1034 ; de l'incendie du temple de la Paix et du Palatin, D.C. LXXII, 24, 2 ; Hdn. I, 14, 26. ;

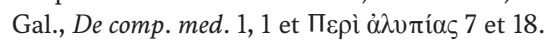

12- Cf. Ov., Tr. III, 1, 6072 ; Pont. I, 1, 56.
13- À propos de ces divers autodafés, cf. D.C. LVI, 27, 1 ; LVII, 24, 34 ; Sen., Contr. X, praef. 3 ; Sen., Consol. ad M., 1, 3 ; Suet., Cal. 16, 2 ; Tac., Ann. IV, 35 ; XIV, 50, 1 ; Ag. 2, 1.

14- Suet., Tib. 70, 4

15- Ov., Tr. III, 1, 63 ; Suet., Tib. 70, 4.

16- Cf. Boudon-Millot, V., «Un traité perdu de Galien miraculeusement retrouvé, le Sur l'inutilité de se chagriner : texte grec et traduction française ", in Boudon-Millot, V., Guardasole, A., Magdelaine, C. (éd.), La science médicale antique. Nouveaux regards. Études réunies en l'honneur de Jacques Jouanna, Paris, Beauchesne, 2008, p. 73-123

17- Cf. Gell. XVI, 8, 2; V, 21, 9 ; XI, 17, 1. Sur la présence d'archives d'État dans le palais impérial, cf. D.C. LXXII, 24, 2 ; Gal., De comp. med. 1,1 .

18- Cf. Houston, G., « The Slave and Freeman Personnel of Public Libraries in Ancient Rome ", in TAPhA 132 (12), 2002, p. 139-176 ; Roman Libraries, p. 217-252.

19- Cf. Gell. XI, 17 ; XIII, 20, 1 ; Fronto, Ad M. Caes. IV, 5.

20- SEG XXI, 500

21- Fronto, Ad M. Caes. IV, 5, 2

22- Gell. XIX, 5, 14 